\title{
Cardiotocogram Data Classification using Random Forest based Machine Learning Algorithm
}

\author{
M. M. Imran Molla ${ }^{1}$, Julakha Jahan Jui ${ }^{2}$, Bifta Sama Bari ${ }^{2}$, Mamunur Rashid ${ }^{2}$ and Md \\ Jahid $\operatorname{Hasan}^{3}$ \\ ${ }^{1}$ Faculty of Computer Science and Engineering, Khwaja Yunus Ali University, Bangladesh. \\ ${ }^{2}$ Faculty of Electrical \& Electronics Engineering, Universiti Malaysia Pahang, 26600 Pekan, \\ Pahang, Malaysia. \\ ${ }^{3}$ Faculty of Mechanical \& Manufacturing Engineering, Universiti Malaysia Pahang, 26600 \\ Pekan, Pahang, Malaysia. \\ mmimranmolla@gmail.com, julakha.ump@gmail.com, \\ biftasama_120@yahoo.com, mamun110218@gmail.com, \\ sawikotegmail.com
}

\begin{abstract}
The Cardiotocography is the most broadly utilized technique in obstetrics practice to monitor fetal health condition. The foremost motive of monitoring is to detect the fetal hypoxia at early stage. This modality is also widely used to record fetal heart rate and uterine activity. The exact analysis of cardiotocograms is critical for further treatment. In this manner, fetal state evaluation utilizing machine learning technique using cardiotocogram data has achieved significant attention. In this paper, we implement a model based CTG data classification system utilizing a supervised Random Forest (RF) which can classify the CTG data based on its training data. As per the showed up results, the overall performance of the supervised machine learning based classification approach provided significant performance. In this study, Precision, Recall, F-Score and Rand Index has been employed as the metric to evaluate the performance. It was found that, the RF based classifier could identify normal, suspicious and pathologic condition, from the nature of CTG data with $94.8 \%$ accuracy.
\end{abstract}

Keywords: Fetal heart rate, Random Forest classifier, Cardiotocography.

\section{Introduction}

Cardiotocography is a strategy that is utilized to screen fetal health condition during pregnancy. A cardiotocogram (CTG) comprises of two signals, to be specific, the fetal heart rate (FHR) as well as uterine activity (UA). The identification of fetal hypoxia at early stage is the target for CTG monitoring. Further examinations for fetal condition may be performed or the baby is delivered by a surgical strategy. A standardized nomenclature has been embraced to peruse the cardiotocographs [1]. It incorporates baseline fetal heart rate (110 to 160 beats/minute), uterine activity, baseline FHR variability ( 5 to 25 beats/ minute above and below the stable FHR baseline), periods of decreased and increased FHR variability and existence of any acceleration or deceleration [2]. It is conceivable to recognize the fetal hypoxia (lack of oxygen normally in the range of 1 to 5\%) by observing FHR. The possibility of being disabling of the newborn baby gets to be high and, in some cases, it may lead to the death if fetal hypoxia is prolonged. Consequently, it is essential to detect abnormal FHR patterns and take suitable actions for evading prenatal morbidity as well as mortality [3,4]. Cardiotocography can be utilized to examine the fetus health condition, normoxia [5] 
28. Chen, X., Ye, Y., Xu, X. and Huang, J.Z.: A feature group weighting method for subspace clustering of high-dimensional data. Patt0 Recognition, 45(1), 434-446, 2012.

29. Zhou, J. and Sun, S.: Active learning of Gaussian processes with manifoldpreserving graph reduction.Neural Computing and Application 25(7-8), 16151625, 2014.

30. Cruz, R.M., Sabourin, R., Cavalcanti, G.D. and Ren, T.I.: META-DES: A dynamic ensemble selection framework using meta-learning. Pattern Recognition48(5), 1925-1935, 2015.

31. UCI Machine Learning Repository,http://archive.ics.uci.edu/ml/datasets/Cardiotocography.

32. Ayres-de Campos, D., Bernardes, J., Garrido, A., Marques-de-Sá, J., Pereira-Leite, L.: SisPorto 2.0: a program for automated analysis of cardiotocograms.Journal of Maternal Fetal Medicine 9(5), 311-318 (2000).

33. Patel, S.: Chapter 5: Random Forest Classifier. May. 18, (2017). 